\title{
Perfil dos Pacientes com Diagnóstico de Câncer de Cabeça e Pescoço atendidos em Hospital Especializado de Recife - PE, no ano de 2014
}

\author{
Carlos Pereira de Brito Neves ${ }^{1}$; André Pereira Gomes Raposo ${ }^{2}$; \\ Phelipe Cunha Bezerra ${ }^{3}$
}

\begin{abstract}
Resumo: Os cânceres de cabeça e pescoço tem crescido no mundo inteiro. São cerca de 200 mil pessoas por ano. Em termos de localização, os cânceres de cavidade oral, possuem uma incidência média de 13.470 novos casos novos por cada 100 mil habitantes, somente no Brasil. O objetivo do presente estudo foi analisar o perfil dos pacientes que tiveram câncer de cabeça e pescoço e que foram atendidos no hospital de câncer de Pernambuco no ano de 2014. Este estudo analisou 560 pacientes no ano de 2014, em um hospital especializado de Recife, Pernambuco, Brasil. As variáveis analisadas incluíram sexo, idade, histórico de consumo de álcool e cigarro, diagnóstico, localização do tumor, o tipo do tumor, primeiro tratamento recebido e desfecho deste tratamento. Os resultados demonstraram que a amostra foi predominantemente de homens $(76,1 \%)$, de cor da pele parda $(68,6 \%)$, tabagistas $(59,5 \%)$, etilistas $(51,4 \%)$ com idades entre 50 e 59 anos $(32,5 \%)$, sendo que $61,2 \%$ possui nível de instrução fundamental. O local do tumor mais prevalente foi C02.9 (17,3\%), o tipo histológico 807013 $(80,5 \%)$ e o local da metástase mais prevalente foi C77 em 85,5\% dos casos. Conclusões: Este estudo reafirma achados da literatura sobre a temática, bem como contribui para um maior conhecimento sobre as características dos pacientes atendidos num hospital especializado em Recife. Certamente uma grande contribuição para que se possa traçar políticas públicas mais adequadas para este tipo de doença.
\end{abstract}

Palavras-chave: Câncer, neoplasias de cabeça e pescoço, álcool e tabaco.

\section{Patients Profile with Cancer Diagnosis of Head and Neck at a Specialized Hospital of Recife - Pernambuco, in the Year of 2014}

\begin{abstract}
Head and neck cancers have been growing all over the world. There are about 200 thousand people a year. In terms of location, oral cavity cancers have an average incidence of 13,470 new cases per 100,000 inhabitants, in Brazil alone. The objective of the present study was to analyze the profile of patients who had head and neck cancer who were treated at the Pernambuco Cancer Hospital in the year 2014. This study analyzed 560 patients in the year 2014 at a specialized hospital in Recife, Pernambuco Brazil. The analyzed variables included sex, age, history of alcohol and cigarette consumption, diagnosis, tumor location, tumor type, first treatment received and outcome of this treatment. The results showed that the sample was predominantly male $(76.1 \%)$, brown skin color $(68.6 \%)$, smokers $(59.5 \%)$, and alcoholics $(51.4 \%)$ aged $50-59(32.5 \%)$, with $61.2 \%$ having a fundamental education level. The most prevalent tumor site was C02.9 (17.3\%), histological type $807013(80.5 \%)$ and the most prevalent site of metastasis was C77 in $85.5 \%$ of the cases. Conclusions: This study reaffirms findings from the literature on the subject, as well as contributes to a better knowledge about the characteristics of patients attended at a specialized hospital in Recife. Certainly a great contribution to the development of public policies more suitable for this type of disease.
\end{abstract}

Keywords: Cancer, head and neck neoplasias, alcohol and tobacco.

\footnotetext{
${ }^{1}$ Médico pela Faculdade de Medicina de Juazeiro do Norte. Residências Médicas em Cirurgia geral(Universidade Federal do Ceará) e, Cirurgia de Cabeça e pescoço (Hospital de Câncer de Pernambuco). Precptor da Residência Médica em cirurgia geral da Universidade Federal do Cariri. Contato: cpbneves@ hotmail.com;

2 Médico pela Universidade de Pernambuco. Residência em Cirurgia Geral (Hospital Agamenom Magalhães, Recife-PE. Residência em Cirurgia de Cabeça e Pescoço pelo AC Camargo Cancer Center - SP. Fellow do Istituto Europeo di Oncologia, Fondazione Umberto Veronesi, Milano-Italia. Titular da Cirurgia de Cabeça e Pescoço: Hospital de Câncer de Pernambuco; Real Hospital Português de Beneficência em Pernambuco e Hospital dos Servidores do Estado de Pernambuco;

3 Médico, Cirurgião Geral e Cirurgião de Cabeça e Pescoço. Médico-assistente em Cirurgia de Cabeça e Pescoço do Hospital de Câncer de Pernambuco, do Hospital Universitário Oswaldo Cruz e, do Hospital das Clínicas. Mestre em Ciências da Saúde pela Universidade de Pernambuco - UPE.
} 


\section{Introdução}

Câncer de cabeça e pescoço é descrito como uma espécie de tumor maligno do trato digestivo superior. Trata-se de uma região que inclui a cavidade oral, a faringe e a laringe. Os carcinomas de cabeça e pescoço são referidos na literatura como cânceres orais, que podem aparecer, principalmente na mucosa da boca, incluindo-se aí a língua, lábios, assoalho bucal, e o palato duro, além da faringe (nestes casos inclui-se a hipofaringe, orofaringe e nasofaringe).

Segundo Dedivitis et. al. (2004), em torno de 40\% dos cânceres de cabeça e pescoço aparecem na cavidade oral, sendo que $15 \%$ surgem na faringe e, $25 \%$ na laringe. Em percentuais menores na tireóide e glândulas salivares (DOBROSSY , 2005). Histologicamente o tipo que se configura com mais frequência é o carcinoma espinocelular. Este tende a apresentar-se em cerca de 90\% dos casos (DEDIVITIS et. al. (2004).

Trata-se de uma doença que responde por inúmeros óbitos no mundo todo, constituindo-se na sexta causa de morte por câncer. Em todo o mundo, surgem, pelo menos 200 mil novos casos de cânceres de cabeça e pescoço todos os anos (WALKER; BOEY; MCDONALD. 2003). No Brasil, são estimados cerca de mais de 13 mil e quatrocentos casos novos de cânceres na cavidade oral por cada 100 mil habitantes. Desses, em torno de mais de dez mil casos acometem pessoas do sexo masculino e, mais de três mil e quatrocentos casos incidem em pessoas do sexo feminino (INSTITUTO NACIONAL DO CÂNCER , 2005).

A incidência de cânceres na região bucal, no país, tem representado cerca de $2 \%$ de todos os casos, mostrando-se uma das mais altas em todo o mundo. Na América Latina apresenta grande expressividade (WUNSCH , 2004). Relativamente a taxa de mortalidade, estima-se que acomete cerca de mais de 12 mil pessoas a cada ano (6), com uma sobre vida de pouco mais de 40 ou 50\% dos pacientes que são diagnosticados (FRANCESHI; BIDOLI; HERRERO e MUNOZ, 2000; ZENDER e PETRUZZELLI, 2005).

A incidência deste tipo de câncer parece aumentar com a idade. Estudos na Europa demonstraram que em $98 \%$ dos casos, os pacientes possuem idade maior que 40 anos (DEDIVITIS et. al. , 2004) . Trata-se de um tipo raro de tumor em pacientes mais jovens. Isto é, em pessoas com menos de 40 anos de idade, sua incidência é de apenas 4 a $6 \%$. Porém parece vir aumentando em muitos países (IAMARROM et al., 2004). Os cânceres nesta faixa 
de idade ainda são pouco conhecidos (DEDIVITIS et. al. , 2004; ZENDER e PETRUZZELLI, 2005; KIM; HONG ; KHURI, 2002).

O álcool e o tabaco são apontados como fatores de risco para os cânceres de cabeça e pescoço (LLEWELLY et. al., 2004). Este atinge mais os pacientes do sexo masculino, porém tem se observado uma maior incidência entre pessoas do sexo feminino (BRADLEY e RAGHAVAN, 2004)

Objetivou-se uma analise do perfil dos pacientes que tiveram câncer de cabeça e pescoço e que foram atendidos no Hospital de Câncer de Pernambuco no ano de 2014. Adicionalmente acreditou-se importante: a) Caracterizar a amostra dos pacientes acometidos de Câncer de cabeça e pescoço, de acordo com os prontuários de um hospital especializado em Recife - Pernambuco, que fizeram parte do estudo, por sexo, idade e escolaridade; b) Identificar o local do tumor cancerígeno no período da pesquisa, no referido hospital; e, c) Estratificar o tipo de tumor cancerígeno quanto a sua gravidade, nos casos da amostra; d) Conhecer o tipo de tratamento e situação do óbito.

\section{Método do Estudo}

Trata-se de um estudo retrospectivo elaborado através de uma análise de prontuários médicos dos pacientes acometidos por cânceres de cabeça e pescoço, que foram atendidos no Hospital de Câncer de Pernambuco, no ano de 2014.

Foram localizados os pacientes com tumores, que foram classificados segundo sua localização anatômica, notadamente aqueles nas cavidades orais, faringe e laringe. Os outros locais incluíram os lábios, a língua, a mucosa oral, palato, gengivas e palato duro.

Segundo Lee (2003), as regiões da faringe são três (orofaringe, onde encontra-se o palato mole, úvula, tonsilas e as paredes posteriores e laterais da orofaringe; a hipofaringe, que compreende os seios piriformes, as paredes hipofaringeanas, e as regiões pós-cricóides e não pós-cricóides. E, a nasofaringe e suas paredes laterais e coanas. No caso da laringe, esta compreende a supraglote, o glote e o subglote.

Fizeram parte deste estudo 560 pacientes de ambos os sexos. 
A análise dos dados partiu da construção de um banco de dados na planilha eletrônica da Microsoft Excel, que posteriormente foi exportada para o programa SPSS, versão 18, onde foi realizada a análise. Para descrever o perfil pessoal dos pacientes avaliados, as variáveis que foram analisadas, incluíram sexo, idade, escolaridade, local do tumor, o histórico de consumo de álcool e cigarro, o diagnóstico realizado no hospital, o tipo histológico do tumor, o local de metástase, primeiro tratamento recebido pelo paciente e o desfecho do tratamento.

Foram calculadas as devidas frequências e percentuais, bem como construídas as respectivas distribuições dos dados. Ainda, foi aplicado o teste Qui-quadrado de comparação de proporção para avaliar a significância das diferenças entre os percentuais avaliados. Todas as conclusões foram tiradas considerando o nível de significância de 5\%.

\section{Resultados}

\section{Perfil dos Pacientes}

Na tabela 1 temos a distribuição dos dados de identificação do paciente. Verifica-se que a maioria dos é do sexo masculino $(76,1 \%)$, possui idade de 50 a 59 anos $(32,5 \%)$, é da cor parda $(68,6 \%)$, estudou até o ensino fundamental $(61,2 \%)$ e é casado $(38,8 \%)$. O tese de comparação de proporção foi significativa em todos os fatores avaliados ( $\mathrm{p}$-valor < 0,001), indicando que o perfil descrito é significativamente o mais prevalente entre o pacientes em estudo.

Tabela 1. Distribuição dos dados de identificação do paciente.

\begin{tabular}{lccc}
\hline \multicolumn{1}{c}{ Fator avaliado } & n & \% & p-valor \\
\hline Sexo & & & \\
$\quad$ Masculino & 426 & 76,1 & $<0,001$ \\
Feminino & 134 & 23,9 & \\
\hline Idade & & & \\
Menos de 40 anos & 25 & 4,5 & \\
40 a 49 anos & 63 & 11,3 & \\
50 a 59 anos & 182 & 32,5 & $<0,001$ \\
60 a 69 anos & 149 & 26,6 & \\
70 a 79 anos & 102 & 18,2 & \\
80 ou mais anos & 39 & 7,0 & \\
\hline
\end{tabular}




\begin{tabular}{lccc}
\hline Raça/Cor & & & \\
Branca & 135 & 24,1 & \\
Preta & 41 & 7,3 & $<0,001$ \\
Parda & 384 & 68,6 & \\
\hline Grau de instrução & & & \\
Nenhuma & 142 & 28,2 & \\
Fundamental & 308 & 61,2 & $<0,001$ \\
Médio & 44 & 8,7 & \\
Superior & 9 & 1,8 & \\
Estado civil & & & \\
Solteiro & 203 & 36,4 & \\
Casado & 216 & 38,8 & \\
$\quad$ Viúvo & 73 & 13,1 & $<0,001$ \\
Separado judicialmente & 37 & 6,6 & \\
União consensual & 28 & 5,0 & \\
1p-valor do teste Qui-quadrado para comparação de proporção \\
(se p-valor < 0,05 as proporções diferem).
\end{tabular}

Na tabela 2 temos a distribuição do histórico do habito de consumo da bebida alcoólica e do cigarro. Observa-se que a maioria dos pacientes do serviço são usuários de álcool $(51,4 \%)$ e de cigarro $(59,5 \%)$. O teste de comparação de proporção nos dois fatores avaliados (p-valor < 0,001), indicando que o número de pacientes usuários de álcool e de cigarro é significativamente maior do que o número de não usuários.

Tabela 2. Distribuição do histórico do uso de alcool e cigarro.

\begin{tabular}{ccc}
\hline \multirow{2}{*}{ Histórico do uso } & \multicolumn{2}{c}{ Item avaliado } \\
\cline { 2 - 3 } & Álcool & Cigarro \\
\hline Nunca & $99(23,0 \%)$ & $58(11,6 \%)$ \\
Ex-consumidor & $110(25,6 \%)$ & $145(28,9 \%)$ \\
Sim & $221(51,4 \%)$ & $298(59,5 \%)$ \\
\hline p-valor & $<0,001$ & $<0,001$ \\
\hline 1p-valor do teste & Qui-quadrado para & comparação \\
proporção (se p-valor $<0,05$ as proporções diferem).
\end{tabular}

Na tabela 3 temos a caracterização do diagnóstico dos pacientes. Verifica-se que na maioria dos casos o diagnóstico foi baseado na histologia do tumor $(91,8 \%)$, o paciente não possui informações sobre histórico familiar de câncer $(60,9 \%)$ e não apresenta mais de um tumor $(97,5 \%)$. Assim como nos últimos fatores avaliados, o teste de comparação de 
Id on Line Revista Multidisciplinar e de Psicoloqia

Id on Line Multidisciplinary and Psycology Journal

proporção não foi significativo (p-valor $<0,001$ ), indicando que o perfil descrito é relevantemente o mais presente no grupo de pacientes avaliados.

Tabela 3. Caracterização do diagnóstico.

\begin{tabular}{lccc}
\hline \multicolumn{1}{c}{ Fator avaliado } & N & $\%$ & p-valor \\
\hline Base mais importante & & & \\
para o diagnóstico & & & \\
Exame com imagem & 2 & 0,4 & \\
Citologia & 12 & 2,1 & $<0,001$ \\
Histologia da metástase & 32 & 5,7 & \\
Histologia do tumor & 514 & 91,8 & \\
\hline Histórico familiar & & & \\
Sim & 83 & 14,8 & \\
Não & 136 & 24,3 & $<0,001$ \\
Sem informação & 341 & 60,9 & \\
\hline Mais de um tumor & & & \\
Não & 546 & 97,5 & $<0,001$ \\
$\quad$ Sim & 14 & 2,5 & \\
1p-valor do teste Qui-quadrado para comparação de proporção \\
(se p-valor < 0,05 as proporções diferem).
\end{tabular}

\section{Localização e tipo histológico do Tumor}

Na tabela 4 temos a distribuição do local do tumor. Verifica-se que o local mais acometido por esta doença é o C02.9 (17,3\%), seguido do C32.9 (16,6\%) e C10.9 (12,0\%). Ainda, os locais menos acometidos são: C00.4, C02.8, C08.0, C09.1, C10.3 e C30.0 (ambos com $0,2 \%$ dos casos).

Tabela 4. Distribuição do local do tumor.

\begin{tabular}{ccc}
\hline Local do tumor & $\mathbf{N}$ & $\mathbf{\%}$ \\
\hline C02.9 & 97 & 17,3 \\
C 32.9 & 93 & 16,6 \\
C10.9 & 67 & 12,0 \\
C 32.0 & 29 & 5,2 \\
C 32.1 & 29 & 5,2 \\
C04.9 & 28 & 5,0 \\
C05.9 & 25 & 4,5 \\
C06.9 & 21 & 3,8 \\
C01.9 & 19 & 3,4 \\
C07.9 & 19 & 3,4 \\
C09.9 & 17 & 3,0 \\
\hline
\end{tabular}




\begin{tabular}{|ccc|}
\hline C13.9 & 15 & 2,7 \\
\hline C11.9 05.1 & 13 & 2,3 \\
\hline C02.1 & 10 & 1,8 \\
\hline C31.0 & 9 & 1,6 \\
\hline C05.0 & 7 & 1,4 \\
\hline C06.2 & 6 & 1,3 \\
\hline C14.0 & 6 & 1,1 \\
\hline C02.4 & 5 & 1,1 \\
\hline C06.0 & 5 & 0,9 \\
\hline C00.1 & 4 & 0,9 \\
\hline C05.2 & 4 & 0,7 \\
\hline C00.0 & 3 & 0,5 \\
\hline C00.9 & 3 & 0,5 \\
\hline C02.0 & 3 & 0,5 \\
\hline C03.1 04.0 & 3 & 0,5 \\
\hline C12.9 & 2 & 0,4 \\
\hline C32.2 & 2 & 0,4 \\
\hline C00.4 & 2 & 0,4 \\
\hline C02.8 & 1 & 0,2 \\
\hline C08.0 & 1 & 0,2 \\
\hline C09.1 & 1 & 0,2 \\
\hline C10.3 & 1 & 0,2 \\
\hline C30.0 & 1 & 0,2 \\
\hline Pesquis 2014 & & 0,2 \\
\hline
\end{tabular}

Fonte: Pesquisa, 2014

Na tabela 5 temos a distribuição do tipo histológico do câncer. Verifica-se que o tipo mais prevalente é o $8070 / 3$ (80,5\%), seguido do $8071 / 3(4,1 \%)$ e $8020 / 3(2,9 \%)$.

Tabela 5. Distribuição do tipo histológico do câncer.

\begin{tabular}{ccc}
\hline Tipo do tumor & $\mathbf{N}$ & \% \\
\hline $8070 / 3$ & 451 & 80,5 \\
$8071 / 3$ & 23 & 4,1 \\
$8020 / 3$ & 16 & 2,9 \\
$8200 / 3$ & 8 & 1,4 \\
$8010 / 3$ & 7 & 1,3 \\
$8070 / 2$ & 7 & 1,3 \\
$8000 / 3$ & 6 & 1,1 \\
$8072 / 3$ & 6 & 1,1 \\
$8430 / 3$ & 6 & 1,1 \\
$8140 / 3$ & 4 & 0,7 \\
$8051 / 3$ & 3 & 0,5 \\
$8076 / 3$ & 3 & 0,5 \\
$8550 / 3$ & 2 & 0,4 \\
\hline
\end{tabular}




\begin{tabular}{|ccc|}
\hline $8980 / 3$ & 2 & 0,4 \\
\hline $9590 / 3$ & 2 & 0,4 \\
\hline $9680 / 3$ & 2 & 0,4 \\
\hline $8010 / 2$ & 1 & 0,2 \\
\hline $8022 / 3$ & 1 & 0,2 \\
\hline $8082 / 3$ & 1 & 0,2 \\
\hline $8310 / 3$ & 1 & 0,2 \\
\hline $8440 / 3$ & 1 & 0,2 \\
\hline $8525 / 3$ & 1 & 0,2 \\
\hline $8562 / 3$ & 1 & 0,2 \\
\hline $8800 / 3$ & 1 & 0,2 \\
\hline $8801 / 3$ & 1 & 0,2 \\
\hline $9591 / 3$ & 1 & 0,2 \\
\hline $9684 / 3$ & 1 & 0,2 \\
\hline $9719 / 3$ & 1 & 0,2 \\
\hline
\end{tabular}

Fonte: Pesquisa, 2014

Na tabela 6 temos a distribuição do local de metástase. Verifica-se que o C77 é o tipo mais prevalente $(85,5 \%)$, seguido do C34 (6,1\%) e o C41 (4,4\%). Os demais locais apresentaram freqüência igual ou inferior a $1,1 \%$.

Tabela 6. Distribuição local de metastase.

\begin{tabular}{ccc}
\hline Local do tumor & $\mathbf{N}$ & $\mathbf{\%}$ \\
\hline C77 & 153 & 85,5 \\
C34 & 11 & 6,1 \\
C41 & 8 & 4,4 \\
C22 & 2 & 1,1 \\
C71 & 2 & 1,1 \\
C15 & 1 & 0,6 \\
C40 & 1 & 0,6 \\
C44 & 1 & 0,6 \\
\hline
\end{tabular}

Fonte: Pesquisa, 2014

\section{Caracterização do tratamento e Situação de óbito}

Na tabela 7 temos a caracterização do primeiro tratamento recebida pelo paciente e da situação de óbito. Verifica-se que a maioria dos paciente receberam nenhum tratamento no hospital no primeiro momento $(28,0 \%)$ ou receberam a radioterapia + quimioterapia $(21,6 \%)$, não apresentavam indícios da doença ao final do primeiro tratamento no hospital (45,2\%), não tiveram óbito ao fim do tratamento $(68,2 \%)$ e, dos que morreram, $89,3 \%$ foi devido à causa primária diagnosticada. 
Tabela 7. Caracterização do primeiro tratamento recebida pelo paciente e da situação de óbito.

\begin{tabular}{|c|c|c|c|}
\hline Fator avaliado & n & $\%$ & p-valor \\
\hline $\begin{array}{llll}\text { Primeiro } & \text { tratamento } & \text { recebido } & \text { no } \\
\text { hospital } & & & \end{array}$ & & & \\
\hline Nenhum & 157 & 28,0 & \\
\hline Cirurgia & 85 & 15,2 & \\
\hline Radioterapia & 73 & 13,0 & \\
\hline Quimioterapia & 20 & 3,6 & \\
\hline Hormonioterapia & 1 & 0,2 & $<0,001$ \\
\hline Cirurgia + Radioterapia & 68 & 12,1 & \\
\hline Cirurgia + Quimioterapia & 4 & 0,7 & \\
\hline Radioterapia + Quimioterapia & 121 & 21,6 & \\
\hline Cirurgia + Radioterapia + Quimioterapia & 31 & 5,5 & \\
\hline
\end{tabular}

\begin{tabular}{|c|c|c|c|}
\hline $\begin{array}{l}\text { Estado da doença ao final do primeiro } \\
\text { tratamento no hospital }\end{array}$ & & & \\
\hline Sem indícios da doença & 157 & 45,2 & \\
\hline Remissão parcial & 19 & 5,5 & \\
\hline Doença estável & 33 & 9,5 & \\
\hline Doença em progressão & 33 & 9,5 & $<0,001$ \\
\hline Suporte terapeutico oncológico & 54 & 15,6 & \\
\hline Óbito & 51 & 14,7 & \\
\hline $\begin{array}{l}\text { Morte do paciente ao fim do } \\
\text { tratamento }\end{array}$ & & & \\
\hline $\begin{array}{l}\text { Sim } \\
\text { Não }\end{array}$ & $\begin{array}{l}178 \\
382\end{array}$ & $\begin{array}{l}31,8 \\
68,2\end{array}$ & $<0,001$ \\
\hline
\end{tabular}

Causa do óbito motivada pela doença primária

Sim

$159 \quad 89,3$

Não

$19 \quad 10,7$

$<0,001$

${ }^{1} \mathrm{p}$-valor do teste Qui-quadrado para comparação de proporção (se p-valor $<$ 0,05 as proporções diferem).

\section{Discussão}

Em nosso estudo foi observada a predominância de indivíduos na faixa etária entre 50 e 59 anos de idade, o que encontra sintonia com resultados do Serviço de oncologia de Cabeça e Pescoço do Hospital Universitário Oswaldo Cruz, em Recife - Pernambuco, que em caso 
semelhante relata uma freqüência de 55,82\% relativamente a este tipo de tumor, nesta faixa de idade (ANTUNES e ANTUNES, 2004).

Em termos do gênero, este estudo corrobora outros já existentes na literatura, onde se evidenciam uma incidência maior de neoplasia de cabeça e pescoço em pessoas do sexo masculino (DEDIVITIS et. al., 2004). Esta doença parece ser mais rara em mulheres (CARVALHO, 2004). Particularmente quando se trata de países em desenvolvimento, há maior incidência em homens (STEWART e KLEIHUES, 2003). Mesmo assim, parece haver um gradativo aumento na incidência entre pessoas do sexo feminino, que parece estar relacionado com mudanças no consumo de álcool e tabaco pelas mulheres (BRADLEY e RAGHAVAN, 2004).

Em estudo desenvolvido no Serviço de Cabeça e Pescoço em Hospital de Heliópolis, houve uma maior incidência deste tipo de câncer em pacientes de cor da pele branca $(85 \%)$ (CARVALHO et. al., 2001). Nosso estudo demonstrou uma maior incidência em pessoas de pele parda. A cor da pele parda é a que tem um maior crescimento no país. É possível que tal resultado se deva a uma realidade local e da Região Nordeste do país, onde a incidência de sol é maior e as pessoas tendem a uma maioria da população com cor da pele parda, em torno de 62,7\% (VIANA, 2007).

No presente estudo, observou-se uma maioria de pacientes que são tabagistas $(51,4 \%)$ e etilistas $(59,5 \%)$. Este achado parece reforçar uma associação entre o consumo de álcool e de cigarro, como contribuindo para o desenvolvimento de cânceres de cabeça e pescoço. Outros estudos têm reforçado tal associação (DOBROSSY, 2005; AHRENDT et. al., 2000; ZAIN, 2001).

O diagnóstico baseado na histologia do tumor $(91,8 \%)$ foi o mais prevalente. As causas principais de diagnóstico tardio são a desinformação, a desconsideração ante os sintomas e, falta de exames preventivos de rotina. Tal situação tende a implementação de tratamentos mais invasivos que tendem a reduzir a qualidade de vida de acometidos por câncer de cabeça e pescoço (SANTOS, CANGUSSU, BATISTA e SANTOS, 2009)

O estudo revelou que $60,9 \%$ dos pacientes não possuíam informações sobre histórico familiar de câncer e não apresenta mais de um tumor (97,5\%).

A influência familiar quanto a desenvolvimento de carcinomas de cabeça e pescoço, pode representar significativa (NEGRI, et. al., 2009). Vários são os fatores genéticos 
envolvidos no metabolismo dos carcinogênicos, associados a risco de Cânceres de cabeça e pescoço, mesmo que, nem sempre serem consistentes(NEGRI, et. al., 2009).

Em relação ao local do tumor, em nosso estudo houve uma prevalência de $\mathrm{C} 02.9$ com 17,3\%, seguido de C32.9 (16,6\%) e C10.9 (12,0\%). Outros estudos epidemiológicos, tem indicado uma ocorrência de $40 \%$ de cânceres de cabeça e pescoço, na cavidade oral (DOBROSSY, 2005). Esta situação parece relacionada a histórico de tabagismo e etilismo nos sujeitos da pesquisa, podendo incrementar entre duas até três vezes o risco deste tipo de doença na cavidade oral (IAMARROM, et. al., 2004; LLEWELLY, et. al., 2004).

Com relação ao tipo histológico do carcinoma, foi mais representativo o tipo 8070/3 (80,5\%), seguido do $8071 / 3(4,1 \%)$ e $8020 / 3$ (2,9\%). Estudo realizado no estado de Pernambuco, observou como mais frequente o carcinoma espinocelular (ANTUNES e ANTUNES, 2004). A literatura também corrobora este tipo de carcinoma, com $90 \%$ de incidência, nos casos de câncer de cabeça e pescoço (CASIGLIA e WOO, 2001).

O local mais prevalente da metástase foi C77 (85,5\%), seguido do C34 $(6,1 \%)$ e do C41 (4,4\%). Os outros locais onde apareceram as metástases, apresentaram frequiência igual ou inferior a $1,1 \%$. Na literatura há referência de uma frequência elevada dos cânceres de cabeça e pescoço já em um estágio avançado (HERCHENHORN e DIAS , 2004). Estudo levado a cabo no Brasil demonstrou diferenças significativas estatisticamente, dessas características, quando foram comparadas a características de pacientes oriundos de instituições estrangeiras (CARVALHO et. al., 2004 )

Quanto ao primeiro tratamento recebido pelo paciente, a literatura refere a cirurgia aberta e a radioterapia externa como principais estratégias no tratamento dos carcinomas (FILHO, SOBRINHO, RAPOPORT, FERREIRA e JULIANO, 2006). O presente estudo observou que não houve nenhum tratamento no hospital, num primeiro momento $(28,0 \%)$ ou receberam a radioterapia + quimioterapia $(21,6 \%)$.

Um estudo com 1010 sujeitos, 528 casos (52,3\%) foram submetidos à cirurgia, 335 $(33,2 \%)$ foram tratados com cirurgia em associação com a radioterapia e, $67(6,6 \%)$ foram tratados com os três procedimentos: cirurgia, quimioterapia e radioterapia (FILHO, SOBRINHO, RAPOPORT, FERREIRA e JULIANO, 2006). 
Em relação à mortalidade, 68,2\% não tiveram óbito e, 89,3\% dos que morreram foi devido à causa primária diagnosticada. Trata-se de uma doença bastante agressiva, com alta taxa de mortalidade (KIM, HONG e KHURI, 2002).

Estes resultados demonstram a necessidade de implementação de programas em educação e saúde, para populações que apresentem maior risco deste tipo de câncer. Outras medidas que venham a minimizar ou atenuar os riscos de cânceres orais ou o desenvolvimento de novos tumores secundários também são bem vindas.

\section{Conclusões}

A análise dos 560 pacientes atendidos no Hospital de Câncer de Pernambuco em 2014, parece estar em acordo com resultados da literatura dados da literatura especializada, que demonstram ser o câncer de cabeça e pescoço mais freqüente em pessoas do sexo masculino, que são tabagistas e com idades entre 50 e 59 anos. A cavidade oral parece mais representativa. Identificar mais precocemente os fatores de risco associados a esta doença, certamente viabilizarão estratégias mais eficazes e a implementação de programas de prevenção adequados à realidade estudada.

\section{Referências}

AHRENDT SA, CHOWN JT, YANG SC, WU L, ZHANG M-J, JEN J SIDRANSKY D. Alcohol consumption and cigarette smoking increase the frequency of p53 mutations in nonsmall cell lung cancer. Cancer Res, v. 60, n.31, p.55-9, 2000.

ANTUNES AA, ANTUNES AP. Estudo retrospectivo e revisão de literatura dos tumores dos lábios: experiência de 28 anos. Rev Bras Cancerologia, v.50, p.295-300, 2004.

BRADLEY PJ, RAGHAVAN U. Cancer presenting in the head and neck during pregnancy. Curr Opin Otolaryngol \& Head Neck Sur, v.12, p.76-81, 2004. 
Id on Line Revista Multidisciplinar e de Psicologia

Id on Line Multidisciplinary and Psycology Journal

CARVALHO AL, BRUVANESH S, SPIRO RH, KOWALSKI LP, SHAH JP. Cancer of the oral cavity: a comparison between institutions in a developing and a developed nation. Head Neck, v. 2, p.31-8, 2004.

CARVALHO MB, LENZI J, LEHN CN, FAVA AS, AMAR A, KANDA JL, WALDER F, MENEZES MB, FRANZI AS, MAGALHÃES MR, CURIONI OA, MARCEL R, SZELIGA S, SOBRINHO J, Rapoport A. Características clínico-epidemiológicas do carcinoma epidermóide de cavidade oral no sexo feminino. Rev. Assoc Med Bras, v. 47, p. 208-14, 2001.

CASIGLIA J, WOO SB. A comprehensive review of oral cancer. Gen Dent, v.49, p.72-82, 2001.

DEDIVITIS RA, FRANÇA CM, MAFRA ACB, GUIMARÃES FT, GUIMARÃES AV. Características clínico-epidemiológicas no carcinoma espinocelular de boca e orofaringe. Rev Bras Otorrinolaringol, v. 70, p. 35-40, 2004.

DOBROSSY L. Epidemiology of head and neck cancer: magnitude of the problem. Cancer and Metastasis Rev , v. 24, p.24:9-17, 2005.

FILHO FSA, SOBRINHO JA, RAPOPORT A, FERREIRA NN, JULIANO Y. Paradigma da disseminação linfática no carcinoma espinocelular da base de língua. Rev Col Bras Cir, v. 33, p. 79-83, 2006.

Franceshi S, Bidoli E, Herrero R, Munoz N. Comparision of cancers of the oral cavity and pharynx worldwide: Etiological clues. Oral Oncol , v.36, p.106-15, 2002.

HERCHENHORN D, DIAS FL. Avanços no tratamento quimioterápico e radioterápico do câncer de cabeça e pescoço. Rev Hosp Clin Fac Med São Paulo, v.59, p.39-46, 2004.

Home Page: INSTITUTO NACIONAL DO CÂNCER. [citado em 2017 Set]. Disponível em http://www.inca.gov.br.

IAMARROM A, PATTANAPORN K, PONGSIRIWET S, WANACHANTARARAK SW, PRAPAYASATOK S, JITTIDECHARAKS S, et al. Analysis of 587 cases of oral squamous cell carcinoma in northern Thailand with a focus on young people. Int $\mathbf{J}$ Oral Maxillofac Surg, v.33, p.84-8, 2004.

Kim ES, Hong WK, Khuri FR. Chemoprevention of aerodigestive tract cancers. Annu Rev Med, v.53, p.223-43, 2002.

LEE KJ. Essential otolaryngology: head e neck surgery. 8th ed. New Haven (Connecticut): McGraw-Hill; 2003. 
Llewelly CD, Linklater K, Bell J, Johnson NW, Warnakulasuriua S. An analysis of risk factors for oral cancer in young people: a case-control study. Oral Oncol, v.40, p.304-13, 2004.

STEWART BW, KLEIHUES P. World Cancer Report, WHO International Agency for Research on Cancer, IARC Press, Lyon, 2003.

VIANA, Larissa. O idioma da mestiçagem. As irmandades de pardos na América Portuguesa. Editora Unicamp. 2007

WALKER DM., BOEY G, MCDONALD LA. The pathology of oral cancer. Pathology, v.35, p.376-83, 2003.

Wunsch V. Epidemiologia do câncer de laringe no Brasil. Sao Paulo Med J, v. 122, p.18894, 2004.

ZAIN RB. Cultural and dietary risk factors of oral cancer and precancer - a brief overview. Oral Oncol, v.37, p.205-10, 2001.

Zender CA, Petruzzelli GJ. Why do patients with head and neck squamous cell carcinoma experience distant metastases: can they be prevented? Curr Opin Otolaryngol Head Neck Surg, v.13, p.101-4, 2005.

SANTOS LCO, CANGUSSU MCT, BATISTA OM, SANTOS JP. Oral Cancer: Population sample of the State of Alagoas at a reference hospital. Braz J Otorhinolaryngol., v.75, n.4, p.524-9, 2009.

Como citar este artigo (Formato ABNT):

NEVES, Carlos P. de B.; RAPOSO, André P. G.; BEZERRA, Phelipe C. Perfil dos Pacientes com Diagnóstico de Câncer de Cabeça e Pescoço atendidos em Hospital Especializado de Recife - PE, no ano de 2014. Id on Line Revista Multidisciplinar e de Psicologia, 2017, vol.11, n.37, p. 685-698. ISSN: 1981-1179.

Recebido: 24/09/2017

Aceito: 27/09/2017 